\title{
Efficient Dehazing Technique for Hazy Images using DCP and WAF
}

\author{
Monika \\ Assistant Professor \\ Monad University, India
}

\author{
Lavi Tyagi \\ Assistant Professor \\ Monad University, India
}

\author{
Rajiv Singh, PhD \\ Associate Professor \\ Monad University, India
}

\begin{abstract}
Attenuation minimizes the contrast and air-light enlarges the whiteness in the captured image. Fog and haze are atmospheric conditions generated by floating particles, degrade the quality of images. Haze removal algorithms have become more beneficial for several vision applications. As we know there is no single technique i.e. accurate for all different kind of problems and circumstances. The existing methods have neglected many issues like noise reduction and non-uniform illumination which will be presented in the output image of the existing haze removal algorithms. These existing approaches either required single image or multiple images for removing haze from an input image. Multiple image of a same scene is required in multiple images based algorithms. So, this requirement is not fulfilled mostly times. So, the area of single image dehazing is an active area in the field of digital image processing. This paper introduced an efficient haze removal method based on Dark Channel Prior (DCP) and Weighted Average Filter (WAF). Refinement of transmission map will be done using WAF, and then image is restored. This proposed algorithm is implemented and tested in MATLAB. The results have shown that the proposed algorithm gives quite effective and quality results.
\end{abstract}

\section{Keywords}

Dark Channel Prior (DCP), Guided Filter, Average Filter, Weighted Average Filter.

\section{INTRODUCTION}

Generally, all images of outdoor scenes are usually full of shadows the dark channels of images will be really dark. Due to fog (air-light), a fogy image is clear and brighter than its original image or image without fog [1]. Haze is constituted of aerosol which is a dispersed system of small particles of dust and water droplets suspended in gas [2]. Fog, haze, and smoke are such phenomena due to atmospheric absorption and scattering. Their radiance received by the captured devices like camera from the scene point is attenuated along the line of sight. Furthermore, the incoming light is blended with the air light [1] — ambient light reflected into the line of sight by atmospheric particles. The degraded images lose their contrast and color fidelity [3]. Limited visibility in haze weather strongly influences the accuracy and the general functions of almost outdoor video surveillance or driver assistance systems [4]. Fog detection and its removal is a very critical task due to its dependency on unknown depth or information such as: weather and lighting conditions [5].

\section{Dark Channel Prior (DCP)}

The acronym of DCP is Dark Channel Prior. It is used (helps in) to remove the haze or fog from the input fogy images. To formally describe this observation, we first define the concept of a dark channel. For an arbitrary image J, its dark channel $\mathrm{J}^{\text {dark }}$ is given by

$$
J^{\text {dark }}(x)=\underset{y \epsilon \Omega(x)}{\min }\left({ }_{c \epsilon\{r, g, b\}}^{\min } J^{c}(y)\right)
$$

\section{Fundamentals Steps of Haze Removal Using Dark} Channel Prior (DCP)

$\begin{aligned} \text { i. } & \text { Estimating the Transmission Map } \\ \text { ii. } & \text { Soft Matting } \\ \text { iii. } & \text { Estimating the Atmospheric Light } \\ \text { iv. } & \text { Recovering the Scene Radiance } \\ \text { v. } & \text { Patch Size[3] }\end{aligned}$

\section{Guided Filter}

Filtering is a technique for modifying or enhancing an image. With the help of filtering, enhancement of certain feature of an image or removal of other unwanted features from an image can be done. There are various filter that are used for enhancement of fogy images like average filter, guided filter, median filter etc. So one of the very popular and efficient filter is guided filter. As the guided filter has the ability of preserving edge of an image in a better manner. Guided filter is derived from local linear model, therefore, it is a local linear function of the guidance image, and structure transference from the guidance image to the filtering output is also allowed. [ISSN 2321-8169 P 542544]. But guided filter provides better results in the process of image enhancement but takes more time as compared to Weighted Average Filter (WAF) [6].

\section{Average Filter and Weighted Average Filter}

The mean or average filter is a simple sliding -window spatial domain filter that replace the center or mid value in the mask or window with the average or mean value of all the pixels or picture values in the window. Mean filtering is an efficient and a very simple and easy method of smoothing images. in the other words, it is the method of reducing the amount of intensity variation between one pixel to next pixel. One of the most important application of average filter is noise removal from noisy image and make it very clear and noise free. There are some potential problems with average filter:

- A single pixel with a very unrepresentative value can significantly affect the average value of all the pixels in its neighborhood.

- When the filter neighborhood straddles an edge, the filter will interpolate new values for pixels on the edge and so will blur that edge. This may be a problem if sharp edges are required in the output.

In the case of WAF (Weighted Average Filter), different values to each pixel of the window or mask are assigned and then replace the centered value of the window with the estimated mean value from the window. Weighted average 
filter provides more efficient and effective results as compare to simple average filter.

\section{Transmission Map}

It is an air-light color vector, and the surface radiance vector at the interaction point of the scene and the real world ray corresponding to the pixel is known as the transmission map [9].

\section{LITERATURE REVIEW}

In this paper the author introduce a technique of haze removal using DCP from the input hazy images. The dark channel prior is a kind of statistics of outdoor haze-free images. It is based on a key observation - most local patches in outdoor haze-free images consists some pixels or picture elements whose intensity values are very low in at least one color channel. Thickness of the haze and recover a high quality haze-free image can be directly estimated by Using this prior information with the haze imaging model. Results of various different hazy images demonstrate the power or strength of the proposed prior. By-product of haze removal provides a high quality depth map [10].

This paper proposed an improved image dehazing algorithm using DCP and Multi-Scale Retinex (MSR). This paper provides improved results lies in automatic and fast acquisition of transmission map of the scene. In this paper author implement Multi-scale Retinex algorithm on the luminance component in $\mathrm{YCbCr}$ space, obtain a pseudo transmission map whose function is similar to the transmission map in traditional approach. By the combination of haze image model and the dark channel prior, a author can be recover a high quality dehaze image. This paper has basically two advantages (i) no user interaction is needed, and (ii) restoring the image much faster while maintaining comparable dehazing performance [11].

In the bad weather condition, the captured picture will become blurred and the color is partial gray and white, due to the effect of atmospheric scattering. This situation is the major drawback with the video surveillance system. In this paper, deeply focus on the physical process of imaging in foggy weather. After full study on the haze removal algorithm of single image over the last decade, authors propose an efficient haze removal algorithm which uses a fast bilateral filter combined with DCP. This algorithm starts with the atmospheric scattering model, estimated transmission map by using dark channel prior, and then combines with grayscale to get refined transmission map by using the efficient bilateral filter. This algorithm saves time as compared with original algorithm. Author also analyzed the reasons why the image is darken after the haze removal using DCP, and proposed the improved transmission map formula to overcome this problem. The results of this proposed algorithm show that this algorithm is feasible because it effectively restores the contrast and color of the scene. Hence, significantly improves the visual effects of the image. Images having large sky region usually prone to distortion when using DCP. So author proposed an algorithm to improve the adaptability of the algorithm [12].

This paper proposed a new haze removal technique HDCP which will integrate improved dark channel prior with histogram equalization to remove the haze from color images and weighted guided filter is used to decrease noise from images. The results have shown that the proposed algorithm has shown quite effective results [13].

\section{PROPOSED METHODOLOGY}

The proposed haze removal algorithm diagrammatically can be representation as shown in Fig. 1.

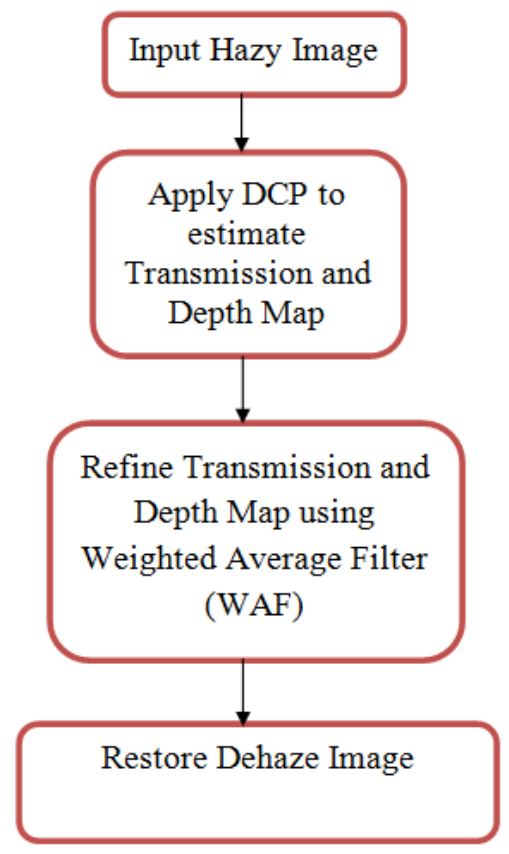

Fig 1: Block Diagram of Proposed Methodology

It includes the estimation of atmospheric light and air-light, transmission estimation, refinement and restoration. Transmission map has been refined using WAF. This filter takes less time as compared with guided filter. Koschmieder's [14] atmospheric scattering model originated the conceptualization of dehazing or defogging algorithms for images. More simply the effect of fog or haze is represented as:

$\mathbf{I}(\mathbf{x})=\mathbf{J}(\mathbf{x}) \mathbf{t}(\mathbf{x})+\mathbf{A}(\mathbf{1}-\mathbf{t}(\mathbf{x}))$

where, $I(x)$ is observed a foggy image, $J(x)$ is desired fogfree image, A denotes the global atmospheric light and $\mathrm{t}(\mathrm{x})$ is the transmission map, the part of light that directly goes towards observer without scattering in the medium.

Airlight is determined by picking up the pixels of the image corresponding to the $0.1 \%$ brightest pixels in the dark channel, and then choosing the one having maximum intensity value.

$$
J^{\text {dark }}(x)=\underset{c \epsilon\{r, g, b\}}{\min }\left(\underset{y \in \Omega(x)}{\min }\left(J^{c}(y)\right)\right)
$$

Transmission estimation is important to measure the thickness of haze or fog. Transmission map, $t(z)$ is the fraction of light that reaches observer without scattering.

$$
\left.t(x)=1-{ }_{c}^{\min }\left(\begin{array}{l}
\min (x) \\
I^{c}(y) \\
A^{c}
\end{array}\right)\right)
$$

Airlight and transmission are sufficient to invert the model and retrieve the original radiance of the scene.

After the estimation of air light and transmission map, refinement is done using WAF to get the quality result.

After refinement restoration of desired haze-free image is done as : 
$J(x)=\frac{I(x)-A}{t(x)}+A$

In order to remove the effect of haze, one must recover J(x). Quantities $\mathbf{A}$ and $t$ are typically unknown, whereas $\mathrm{I}$ is known (input image).

\section{RESULT}

Proposed methodology is implemented in MATLAB. To test this methodology, input images are taken from FRIDA database of fogy road images [16]. In this paper 10 images have been taken and for simplicity a, b, c, d, e, f, g, h , i, j names are given as shown in tables. Table 1 shows the result of these 10 images using DCP and guided filter. After this, Table 2 is given that shows the result of proposed methodology. In this paper, comparison of these two methodologies is done only on the basis of time taken by them. It is so, because proposed algorithm is well suited for real life applications. Table 3 shows the time taken by existing and proposed methodology for the defined 10 images. Comparison shows that this proposed methodology performs better and is best for real life applications ( in bad weather environment conditions like fog, haze, smog etc.).

Table 1: Result using DCP and Guided Filter

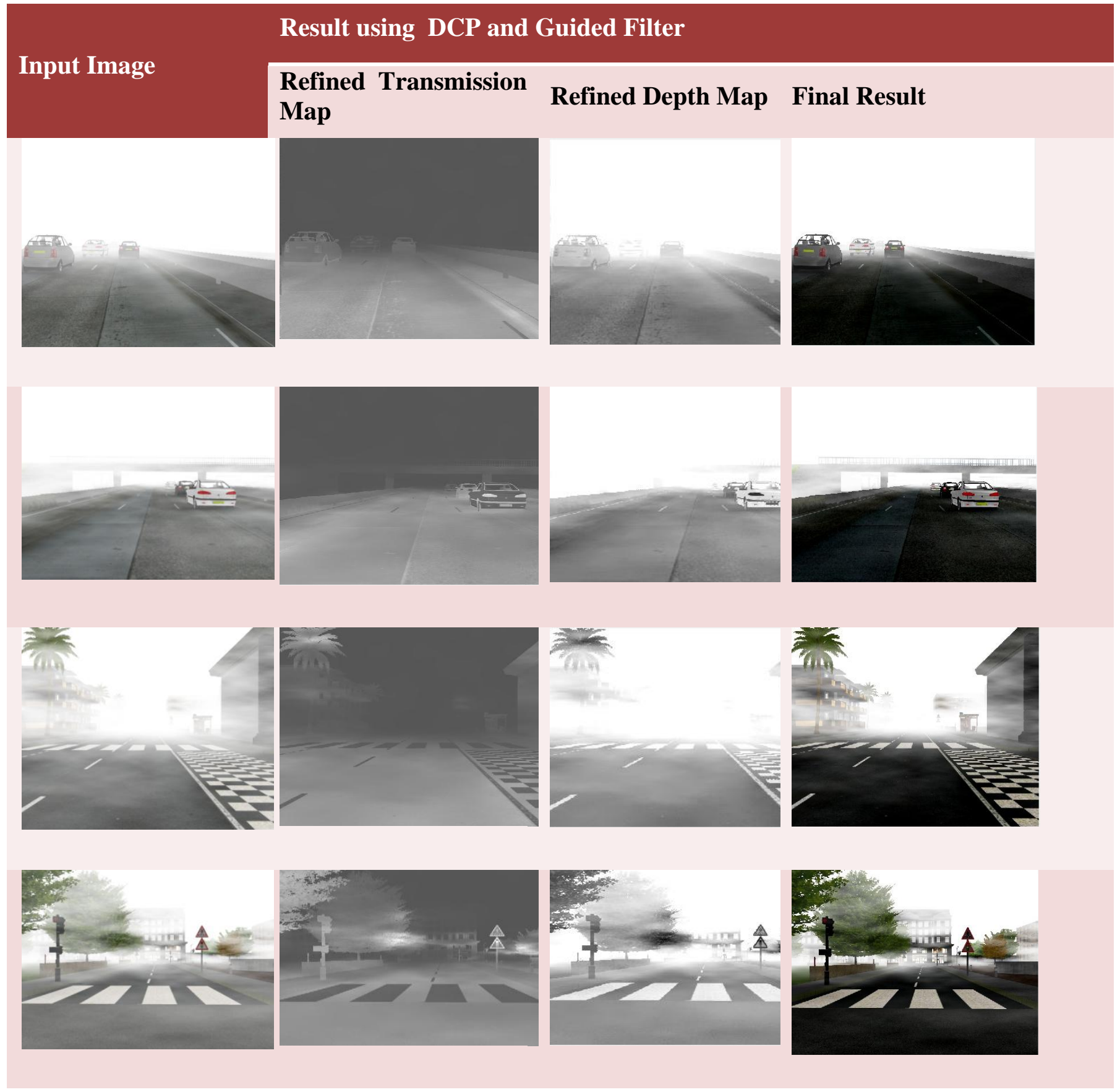



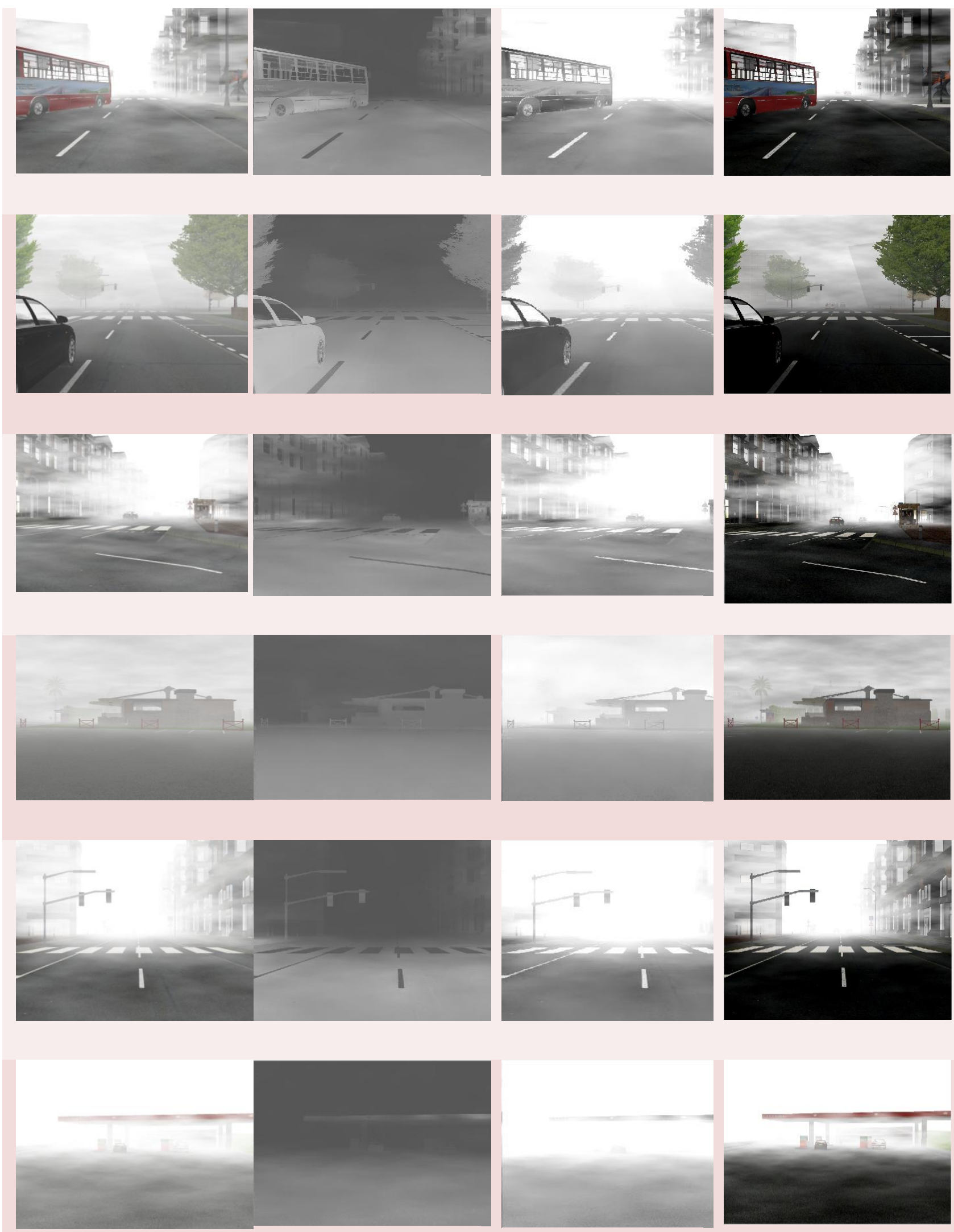
Table 2: Result using Proposed Method

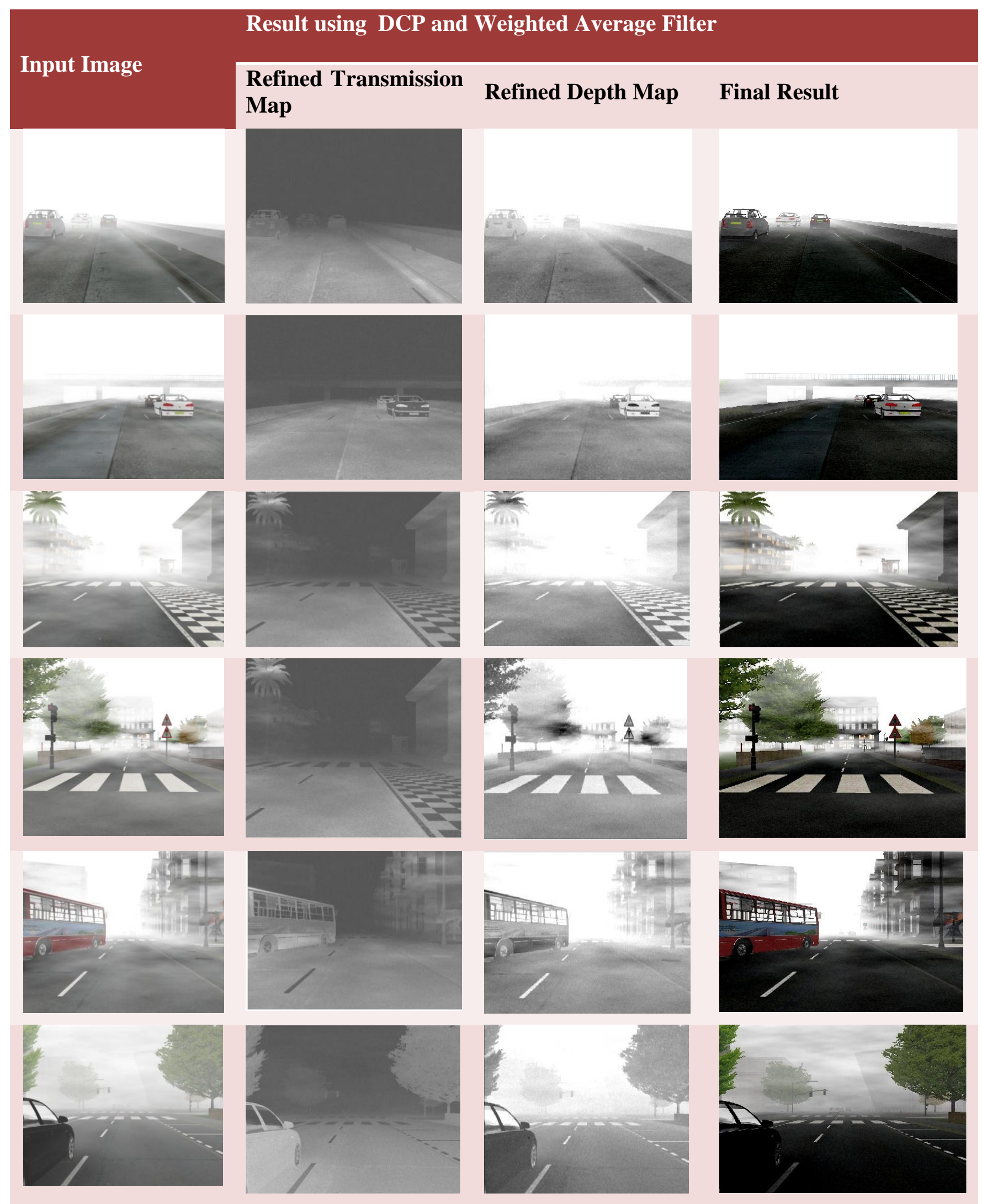



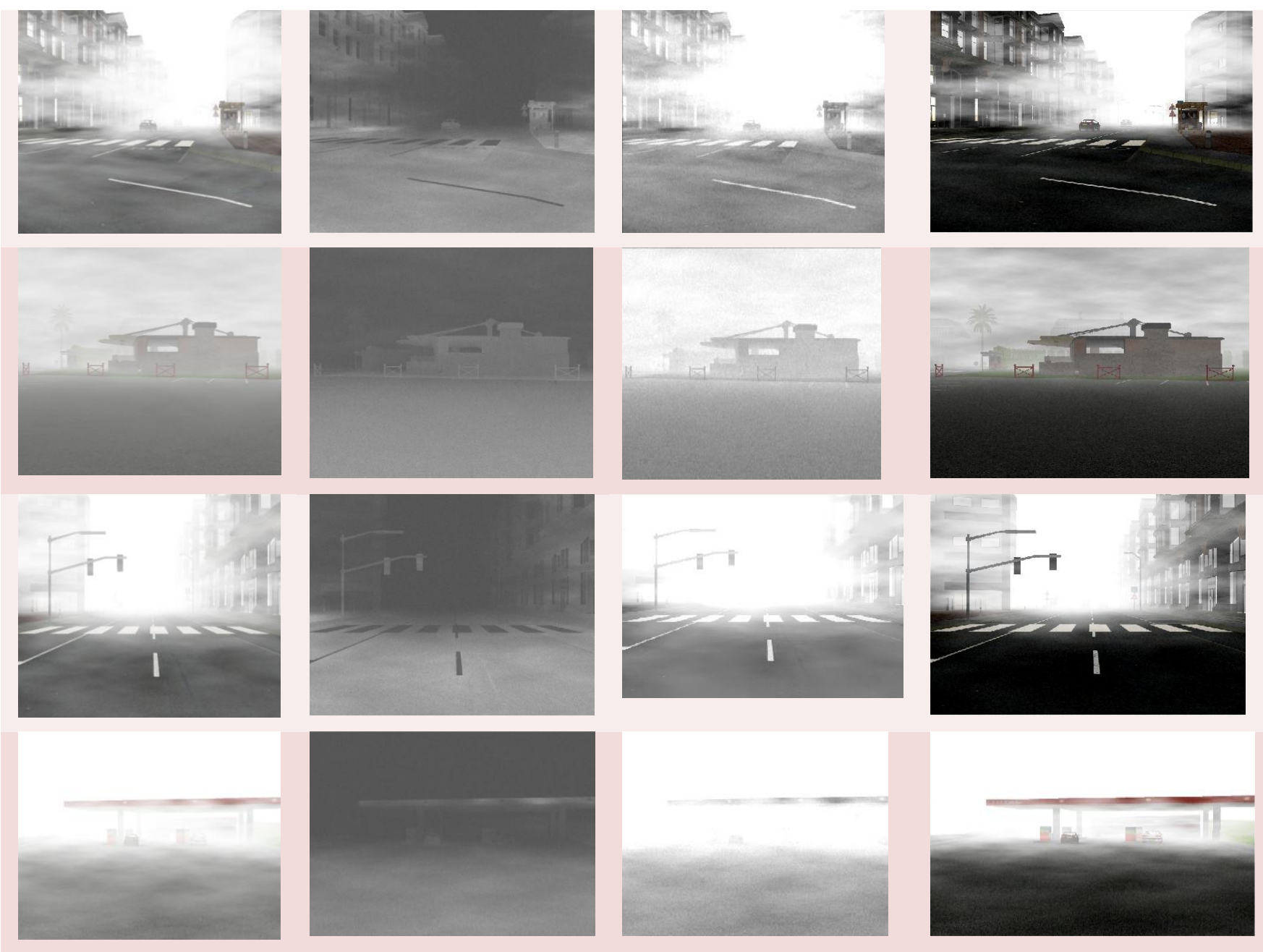

Table 3: Comparison Table

\begin{tabular}{|ccc|}
\hline Tested Images & $\begin{array}{c}\text { Time taken by DCP and } \\
\text { Guided Filter }\end{array}$ & $\begin{array}{c}\text { Time taken by DCP and } \\
\text { Weighted Average Filter }\end{array}$ \\
\hline a.png & 20.201282 & 3.631205 \\
\hline b.png & 1.789569 & 1.516910 \\
\hline c.png & 3.971459 & 1.641539 \\
\hline d.png & 2.148800 & 1.700139 \\
\hline e.png & 1.873951 & 1.529061 \\
\hline f.png & 1.819400 & 1.579768 \\
\hline g.png & 1.825719 & 1.638616 \\
\hline h.png & 1.854381 & 1.577566 \\
\hline i.png & 1.824029 & 1.583092 \\
\hline j.png & 1.750246 & 1.511892 \\
\hline Average & $\mathbf{3 . 9 0 5 8 8 3 6}$ & 1.7909788 \\
\hline
\end{tabular}

\section{CONCLUSION}

It is quite clear from the results shown above, proposed methodology that makes use of DCP and Weighted Average
Filter gives better quality results in terms of minimum time and better appearance, compared with existing ( DCP with 
guided filter). It has wider range of real life applications because it provides efficient results.

\section{FUTURE SCOPE}

This proposed methodology can be used in various real life applications because it provides better quality results efficiently in foggy weather and other bad conditioned weather. It can also be used to identify the criminals from CCTV footage in foggy weather. This technique can be used to minimize road accidents that are occurred due to bad weather conditions (Foggy, Smog etc.), by using this methodology in real life image capturing devices used in vehicles.

\section{REFERENCES}

[1] Saggu, Manpreet Kaur, and Satbir Singh. "A review on various haze removal techniques for image processing." International Journal of Current Engineering and Technology. 5, no. 3 (2015): 15001505.

[2] Narasimhan, Srinivasa G., and Shree K. Nayar. "Vision and the atmosphere." International Journal of Computer Vision 48, no. 3 (2002): 233-254.

[3] He, Kaiming, Jian Sun, and Xiaoou Tang. "Single image haze removal using dark channel prior." IEEE transactions on pattern analysis and machine intelligence 33, no. 12 (2011): 2341-2353.

[4] Mao, Jun, UthaiPhommasak, Shinya Watanabe, and Hiroyuki Shioya. "Detecting foggy images and estimating the haze degree factor." Journal of Computer Science \& Systems Biology 7, no. 6 (2014): 1.

[5] Bronte, Sebastián, Luis M. Bergasa, and Pablo Fernandez Alcantarilla. "Fog detection system based on computer vision techniques." In Intelligent Transportation Systems, 2009. ITSC'09. 12th International IEEE Conference on, pp. 1-6. IEEE, 2009.

[6] He, Kaiming, Jian Sun, and Xiaoou Tang. "Guided image filtering." IEEE transactions on pattern analysis and machine intelligence 35, no. 6 (2013): 1397-1409.
[7] Tomasi, C., Manduchi, R.: Bilateral filtering for gray and color images. In: ICCV (1998).

[8] Levin, Anat, Dani Lischinski, and Yair Weiss. "A closedform solution to natural image matting." IEEE Transactions on Pattern Analysis and Machine Intelligence 30, no. 2 (2008): 228-242.

[9] Guo, Fan, Jin Tang, and Xiaoming Xiao. "Foggy scene rendering based on transmission map estimation." International Journal of Computer Games Technology 2014 (2014): 10.

[10] He, Kaiming, Jian Sun, and Xiaoou Tang. "X.: Single image haze removal using dark channel prior." (2009).

[11] Xie, Bin, Fan Guo, and Zixing Cai. "Improved single image dehazing using dark channel prior and multi-scale Retinex." In Intelligent System Design and Engineering Application (ISDEA), 2010 International Conference on, vol. 1, pp. 848-851. IEEE, 2010.

[12] Xu, Haoran, Jianming Guo, Qing Liu, and Lingli Ye. "Fast image dehazing using improved dark channel prior." In Information Science and Technology (ICIST), 2012 International Conference on, pp. 663-667. IEEE, 2012.

[13] Anupama, Nidhi Singh and Lavi Tyagi. Hybrid Dehazing Technique using IDCP with Histogram Equalization for Color Image. International Journal of Computer Applications 174(1):1-5, September 2017.

[14] W.K. Middleton, Vision through the Atmosphere (1957).

[15] Md. Imtiyaz Anwar, Arun Khosla," Vision enhancement through single image fog removal" Engineering Science and Technology, an International Journal, Volume 20, Issue 3, June 2017, Pages 1075-1083.

[16] http://www.lcpc.fr/english/products/imagedatabases/article/frida-foggy-road-image-database 